\title{
Does Cygnus A harbor a binary super-massive black hole?
}

\author{
Uwe Bach ${ }^{* a}$, Biagina Boccardi ${ }^{a, b}$, Thomas P. Krichbaum ${ }^{a}$, \& Andrei Lobanov ${ }^{a}$ \\ ${ }^{a}$ Max-Planck-Institut für Radioastronommie, Bonn, Germany \\ ${ }^{b}$ Osservatorio di astrofisica e scienza dello spazio di Bologna, Italy \\ E-mail: ubach@mpifr.de, bboccardi@mpifr.de, tkrichbaum@mpifr.de, \\ alobanov@mpifr.de
}

Recently a new radio source was detected with the VLA close to Cygnus A at a distance of about 460 parsec from the center of the AGN. The source is not directly associated with the VLBI core and the jets, but coincides with a compact optical/near-IR source. We detected the new source on sub-parsec scales with EVN observations at $1.3 \mathrm{~cm}$ in March 2017 and $6 \mathrm{~cm}$ in June 2017. It appears nearly point-like and more luminous than any known radio super-nova. The most plausible origin of the emission is a recent onset of activity from a so far not detected secondary super-massive black hole (SMBH). It could be an extreme type of a super-nova, but the enormous luminosity and compactness on sub-parsec scales and the flat spectral index suggest that a secondary SMBH may exist in the immediate surroundings of the central engine in Cygnus A. Here we present our EVN observations and discuss their implications.

14th European VLBI Network Symposium \& Users Meeting (EVN 2018)

8-11 October 2018

Granada, Spain

${ }^{*}$ Speaker. 


\section{Introduction}

Cygnus A is the closest $(z=0.057)$ strong FR II radio galaxy and therefore a key object for detailed studies of its prominent double sided jet and nucleus. Owing to the large inclination of the jet with respect to the observer $\left(>75^{\circ}\right)$, and correspondingly reduced relativistic effects, Cyg A is an ideal candidate for detailed studies of its jet physics, which is thought to be similar to those in the more luminous quasars (e.g. Barthel 1989). Cygnus A has been studied extensively on all scales with radio interformeters and VLBI over the past decades (e.g. Krichbaum et al. 1993; Carilli \& Harris 1996; Krichbaum et al. 1998; Bach 2004; Boccardi et al. 2016b,a)

On the 13th of September 2016, the "Astronomers Telegram" reported the discovery of a new radio source in the vicinity of the nucleus of Cygnus A (Perley et al. 2016). Multi-frequency VLA observations conducted between July 2015 and August 2016 revealed the presence of a point-like object at the location $\mathrm{RA}=19: 59: 28.32345 \mathrm{Dec}=+40: 44: 01.9133$ (J2000), i.e. at a distance of $\sim 460$ parsecs (or $\sim 418$ mas) west/south-west of the CygA radio core. The emission is characterized by a rather flat spectral index $\alpha \sim-0.2$ (with $S_{v} \propto v^{\alpha}$ ), and by a flux density of $\sim 4$ mJy at 10 GHz (Perley et al. 2017).

The source can be classified as a radio transient, since it was not visible (down to a $3 \sigma$ level of $0.7 \mathrm{mJy}$ ) in previous VLA observations from 1989. Our recent VLBI images from 1.6 GHz to 22 GHz obtained during 1998-2006 (Krichbaum et al. 1998; Bach 2004; Boccardi et al. 2016b) do not show this component either. In October 2016 we proposed EVN observations at wavelengths of $18 \mathrm{~cm}, 6 \mathrm{~cm}$, and $1.3 \mathrm{~cm}$ to verify and detect the new feature on parsec scales. The first observation was conducted at a wavelength of $1.3 \mathrm{~cm}$ in March 2017. In June 2017, the remaining other radio bands were observed. Here we present the $1.3 \mathrm{~cm}$ and $6 \mathrm{~cm}$ EVN images and discuss the sub-parsec scale structure of the new source. In the following the new source will be referred to as Cygnus A2.

\section{Observations}

The observations were carried out in the EVN Sessions I and II in 2017 using the full EVN under the project code EB060A $(1.3 \mathrm{~cm}), \mathrm{B}(6 \mathrm{~cm})$, and C $(18 \mathrm{~cm})$. The VLBI array of up to 15 stations (depending on the frequency), consisted of the central European radio-telescopes (Ef, Jb, Mc, $\mathrm{Nt}, \mathrm{On}, \mathrm{Wb}, \mathrm{Ys}$ ), Hh in South Africa, the Chinese stations (Ur, T6), the Russian Kvazar Network ( $\mathrm{Sv}, \mathrm{Zc}, \mathrm{Bd})$, and the Korean KNV antennas $(1.3 \mathrm{~cm}$ only). Observing modes were standard EVN continuum bands using $1 \mathrm{Gbps}$ of recording rate and $128 \mathrm{MHz}$ of bandwidth per polarization (LCP, $\mathrm{RCP}$ ). To obtain a good uv-coverage the observations were spread over 8 hours with a total integration on Cygnus A for about 7 hours. All data were correlated at JIVE. Standard data reduction was applied in AIPS, imaging and model fitting was done in Difmap. One or two stations failed in every observations, but the remaining 12-13 stations including the most sensitive antennas allowed high dynamic range imaging of Cygnus $\mathrm{A}$ and the transient source.

With a separation of 0.4 arcsec the new source is well within the field of view of a global VLBI observation, when taking care of time and bandwidth smearing. To obtain a large enough field of view the data was averaged in time for 4 seconds only and channels were kept at $4 \mathrm{MHz}$ of bandwidth. Estimated by the EVN sensitivity calculator, this should be sufficient for an 0.6 arcsec field of view radius with less than $10 \%$ loss in the response of a point source. 
The resulting map of the $6 \mathrm{~cm}$ observation is presented in Figure 1 showing both sources: Cygnus A with its prominent jets and the new source about $460 \mathrm{pc}$ away ( 1 mas corresponds to $\sim 1.1 \mathrm{pc})$. The inset shows the new source in more detail. Cyg A2 appears mainly compact, but compared to the beam size it is slight broader along the minor axis of the beam and shows a small extension to the south. The peak flux is about $2.2 \mathrm{mJy}$ which corresponds to a $13 \sigma$ detection.

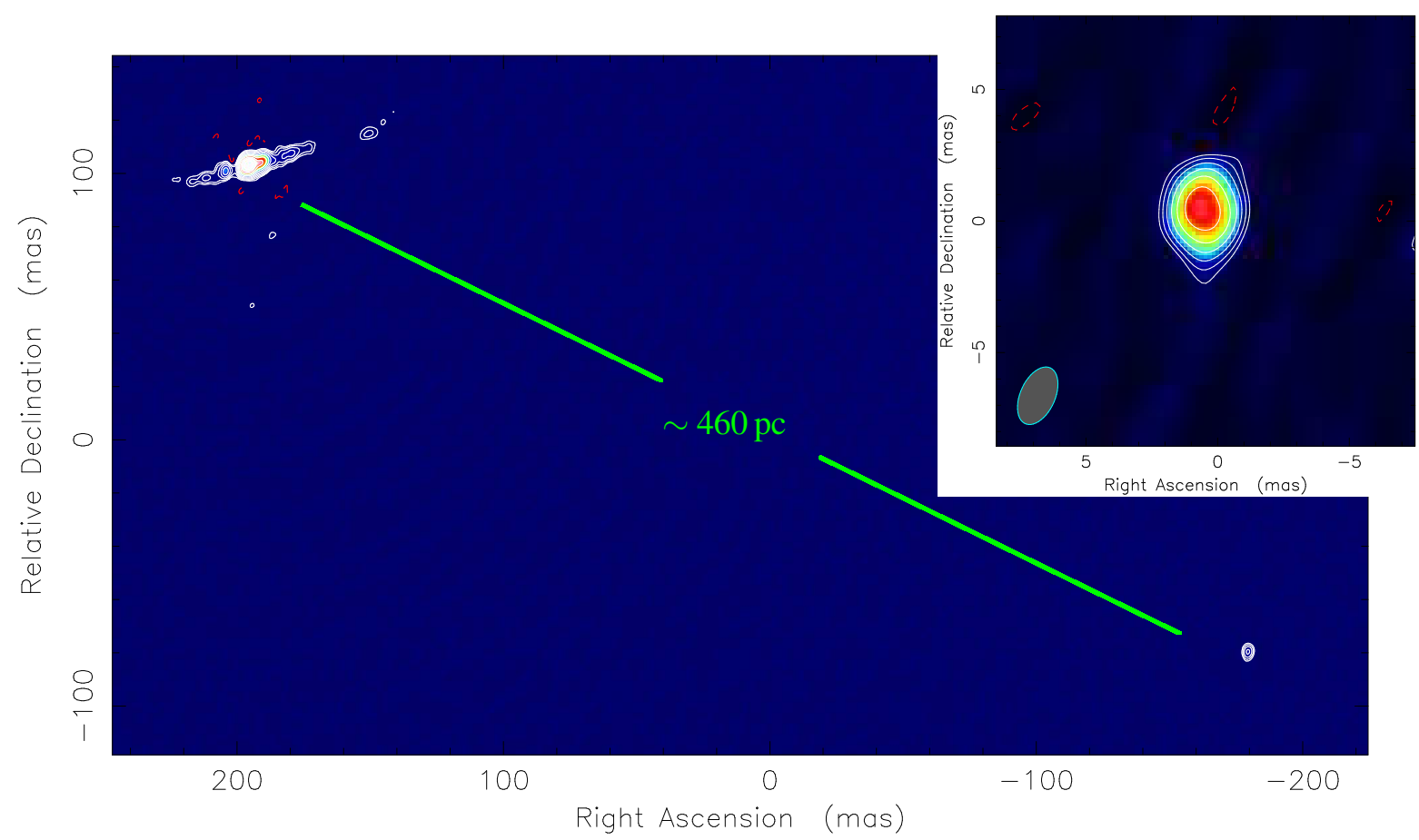

Figure 1: Large scale image at $6 \mathrm{~cm}$ showing Cyg A and Cyg A2 at their total separation of about 460户்c. The map peak is at $300 \mathrm{mJy} / \mathrm{beam}$, the lowest contour starts at $0.35 \mathrm{mJy} / \mathrm{beam}$ and the natural weighted beam is $3.3 \times 2.1$ mas at $-21.3^{\circ}$. The inset in the top-right is a zoom to Cyg A2. The peak flux is $2.2 \mathrm{mJy} / \mathrm{beam}$, the lowest contour starts at $0.3 \mathrm{mJy}$ and the beam is $2.3 \times 1.3 \mathrm{mas}$ at $-24.2^{\circ}$.

In comparison the images at $1.3 \mathrm{~cm}$ are shown in Figure 2. There are still a few issues with the final calibration, visible in the rms pattern of the Cygnus A map (left panel), but comparison with previous maps show similar details and flux density levels. Cygnus A2 (right panel) appears slightly resolved, however the beam in the $1.3 \mathrm{~cm}$ data is also more elongated and the extend is more or less along the beam position angle. The peak flux is about $1.9 \mathrm{mJy}$ which corresponds to a $15 \sigma$ detection. The main image and source properties are listed in Table 1.

The last column of Table 1 lists the brightness temperature obtained from a modelfit of the Cyg A2 component. Given that the source has an SNR of less than 20 and the size of the model fit can easy vary by $20 \%$, the uncertainty on the brightness temperature is likely about a factor of 2 .

When comparing the images of Cygnus A 2 at the two different bands it became obvious that the images are shifted against each other. To properly align the two Cyg A2 images at $6 \mathrm{~cm}$ and $1.3 \mathrm{~cm}$ and, at the same time, obtain a sensible image reference for Cyg A, the $6 \mathrm{~cm}$ image had to be shifted by -2.2 mas in RA and 0.3 mas in DEC. This fits to the values which we found in previous multi-frequency studies of the opacity core shift in Cyg A (Bach 2004, Boccardi, et al. in prep.). The image alignment and spectral index map of Cyg A2 are shown in Figure 3. The peaks of the 
Table 1: The table lists the basic image parameters and a few source details. There are the epoch, frequency, total flux in the image, beam size, peak flux density on Cygnus A, image rms $(\sigma)$, total flux density of the new source, its peak flux density in the image, and the corresponding brightness temperature.

\begin{tabular}{|c|c|c|c|c|c|c|c|c|}
\hline \multirow[b]{2}{*}{ Epoch } & \multirow[b]{2}{*}{$\begin{array}{c}v \\
{[\mathrm{GHz}]}\end{array}$} & \multirow[b]{2}{*}{$\begin{array}{l}S_{\text {tot }} \\
{[\mathrm{Jy}]}\end{array}$} & \multirow[b]{2}{*}{$\begin{array}{c}\text { Beam } \\
{[\text { mas } \times \text { mas }],\left[^{\circ}\right]}\end{array}$} & \multicolumn{2}{|l|}{ Cyg A } & \multicolumn{3}{|c|}{ Cygnus A2 } \\
\hline & & & & $\begin{array}{c}S_{\text {peak }} \\
{\left[\frac{\mathrm{Jy}}{\text { beam }}\right]}\end{array}$ & $\left.\begin{array}{c}\sigma \\
{\left[\frac{\mathrm{mJy}}{\text { beam }}\right]}\end{array}\right]$ & $\begin{array}{c}S_{\text {tot }} \\
{[\mathrm{mJy}]}\end{array}$ & $\begin{array}{c}S_{\text {peak }} \\
{\left[\frac{\mathrm{mJy}}{\text { beam }}\right]}\end{array}$ & $\begin{array}{c}T_{\mathrm{B}} \\
10^{8}[\mathrm{~K}] \\
\end{array}$ \\
\hline & 22.2 & 0.95 & $08 \times 04-15$ & 0.35 & 0.13 & 3.1 & 1.9 & 0.4 \\
\hline 2017.44 & 5.0 & 0.69 & $2.3 \times 1.3,-23$ & 0.19 & 0.16 & 2.6 & 2.2 & 1.6 \\
\hline
\end{tabular}
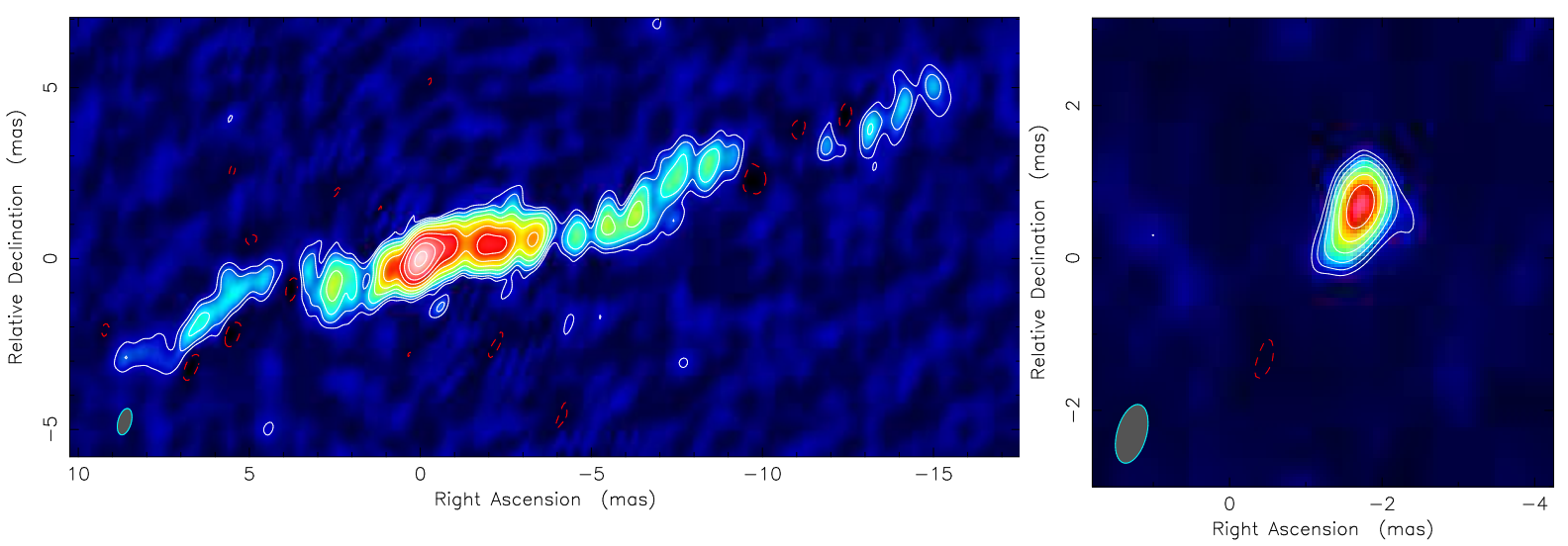

Figure 2: Left: EVN image of Cygnus A at $1.3 \mathrm{~cm}$. The map peak is at $347 \mathrm{mJy} / \mathrm{beam}$, the lowest contour starts at $0.55 \mathrm{mJy} /$ beam and the uniform beam is $0.79 \times 0.39$ mas at $-15.35 \circ$. Right: For this image the map center was shifted to the position of Cyg A2. The peak flux is $1.9 \mathrm{mJy} / \mathrm{beam}$, the lowest contour starts at $0.25 \mathrm{mJy}$ and the beam is $0.79 \times 0.39$ mas at $-15.35 \circ$.

Cyg A2 images still show a small offset, but a further shift would misalign the Cyg A jet images. Therefore this remaining shift could be interpreted as an intrinsic opacity shift in Cyg A2 between the two frequencies. In general, the Cyg A2 feature appears to have a rather flat spectral index.

\section{Results \& Discussion}

The new source, Cyg A2, in the FRII radio galaxy Cygnus A is well detected on VLBI scales. The component appears to be slightly resolved and the VLBI images show a total flux of about $3 \mathrm{mJy}$, with a flat spectral index between wavelengths of $1.3 \mathrm{~cm}$ and $6 \mathrm{~cm}$. This is slightly lower than the flux densities that were measured at the VLA (Perley et al. 2017) and also suggests that the source is slightly resolved on parsec scales. Comparison to a VLBA snapshot observation from November 2016 (Perley et al. 2017) does not show any measurable motion ( $\geq 0.5 \mathrm{mas} / \mathrm{yr}$ ) with respect to the central source Cyg A. Those findings and the fact that the position agrees well with a near-IR source found in Keck and HST images (Canalizo et al. 2003) suggests that the new source belongs to the Cygnus A radio galaxy. Therefore we assume in the following that the source is at the same distance of $D_{\mathrm{L}} \approx 250 \mathrm{Mpc}$.

Model-fitting of the $6 \mathrm{~cm}$ data yields a size of $\sim 0.5 \mathrm{pc}$ and brightness temperature of $T_{\mathrm{B}} \approx$ $1.6 \cdot 10^{8} \mathrm{~K}$, which makes synchrotron emission likely. The flux density of the $1.3 \mathrm{~cm}$ observations 

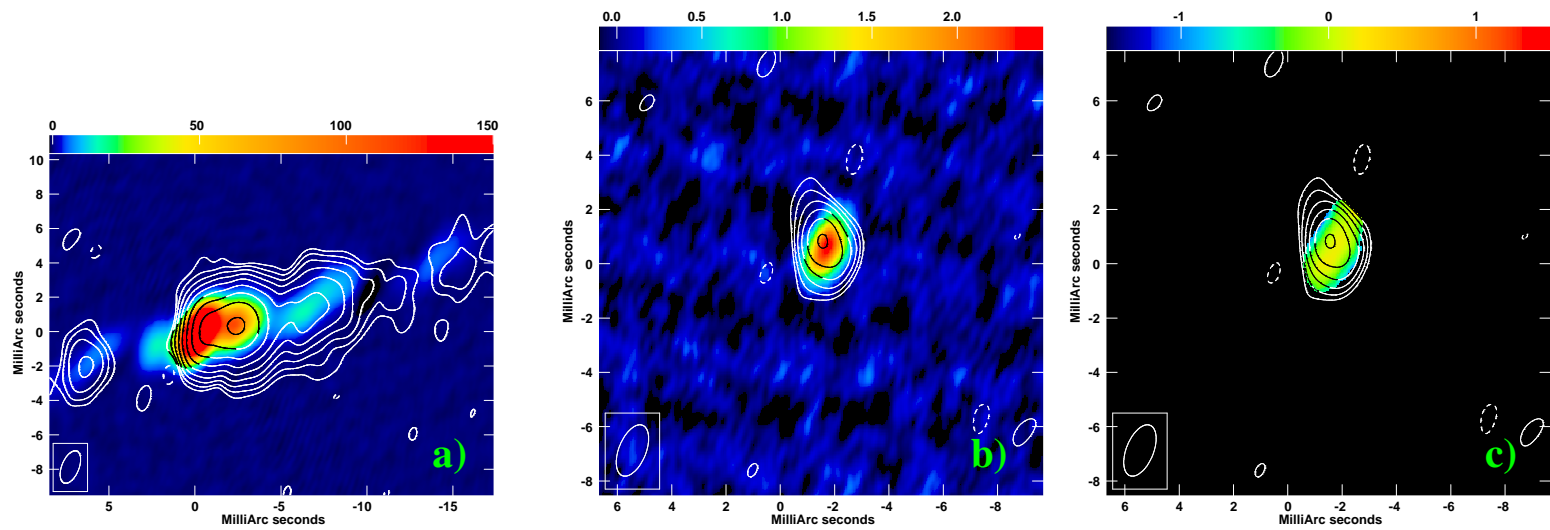

Figure 3: Panels a) \& b): Overlays of the $1.3 \mathrm{~cm}$ image in colors and the $6 \mathrm{~cm}$ image in contours. Both images were convolved with the uniform weighted beam of the $6 \mathrm{~cm}$ data of $2.3 \times 1.3$ mas at $-24.2^{\circ}$. The $6 \mathrm{~cm}$ image was shifted by -2.2 mas in RA and 0.3 mas in DEC. Panel c): Spectral index map of Cygnus A2, $S \propto v^{\alpha}$.

correspond to a radio luminosity of $L_{v} \approx 6 \times 10^{29} \mathrm{erg} / \mathrm{s} / \mathrm{Hz}$ and, assuming that the synchrotron peak is at these radio frequencies, a total luminosity of $L_{v} \approx 10^{40} \mathrm{erg} / \mathrm{s}$. These parameters are quite challenging for all emission processes beside accretion onto a massive black hole. There are supernovae that reach such high luminosity (Perez-Torres et al. 2015) and the star formation rate in Cygnus A could be higher than for a normal galaxy (Privon et al. 2012), but it would be still a very rare event and the most luminous super-nova without a gamma-ray-burst (GRB).

The most plausible origin appears to be accretion onto a secondary massive black hole. Perley et al. (2017) discuss various scenarios. In principle, from Eddington limit arguments the black hole could be as small as $2 \times 10^{3} \mathrm{M}_{\odot}$. If it is the stripped off core from a lower mass galaxy that merged with Cygnus A, it is more likely in the range of $10^{5}-10^{8} \mathrm{M}_{\odot}$. The distribution of gas and dust in the central region suggests that Cyg A underwent at least some minor mergers in the past (e.g. Canalizo et al. 2003). We cannot distinguish yet if emission is due to the onset of activity from a persistent AGN or some kind of abrupt event, like a tidal disruption event (TDE), where a star is disrupted and accreted by a black hole.

Mergers of galaxies are often cited as important triggering mechanisms for activity in radio galaxies and therefore secondary SMBH are expected to be ubiquitous in galaxies, but they remain difficult to detect, with only a few celebrated cases known so far. The discovery that this archetypical powerful FRII radio galaxy may be a binary SMBH could mean that such objects may be more common, and more important, than previously considered. We will continue to monitor this new source and, with time, possible structural changes and flux density variations will tell us more about the nature of Cygnus A2.

\section{References}

Bach, U. 2004, PhD thesis, Rheinische Friedrich-Wilhelms-Universität Bonn

Barthel, P. D. 1989, ApJ, 336, 606

Boccardi, B., Krichbaum, T. P., Bach, U., Bremer, M., \& Zensus, J. A. 2016a, A\&A, 588, L9

Boccardi, B., Krichbaum, T. P., Bach, U., et al. 2016b, A\&A, 585, A33 
Canalizo, G., Max, C., Whysong, D., Antonucci, R., \& Dahm, S. E. 2003, ApJ, 597, 823

Carilli, C. L. \& Harris, D. E. 1996, Cygnus A - Study of a Radio Galaxy (Cambridge: Cambridge University), 76

Krichbaum, T. P., Alef, W., Witzel, A., et al. 1998, A\&A, 329, 873

Krichbaum, T. P., Witzel, A., Graham, D. A., et al. 1993, A\&A, 275, 375

Perez-Torres, M., Alberdi, A., Beswick, R. J., et al. 2015, Advancing Astrophysics with the Square Kilometre Array (AASKA14), 60

Perley, D. A., Perley, R. A., \& Carilli, C. L. 2016, The Astronomer's Telegram, 9495

Perley, D. A., Perley, R. A., Dhawan, V., \& Carilli, C. L. 2017, ApJ, 841, 117

Privon, G. C., Baum, S. A., O’Dea, C. P., et al. 2012, ApJ, 747, 46 